\title{
Analysis of expression sequence tags from a full-length-enriched cDNA library of developing sesame seeds (Sesamum indicum)
}

Tao Ke ${ }^{1,2 \dagger}$, Caihua Dong ${ }^{1 \dagger}$, Han Mao ${ }^{1 \dagger}$, Yingzhong Zhao ${ }^{1}$, Hong Chen ${ }^{1}$, Hongyan Liu', Xuyan Dong ${ }^{1}$, Chaobo Tong ${ }^{1}$ and Shengyi Liu ${ }^{1 *}$

\begin{abstract}
Background: Sesame (Sesamum indicum) is one of the most important oilseed crops with high oil contents and rich nutrient value. However, genetic improvement efforts in sesame could not get benefit from molecular biology technology due to poor DNA and RNA sequence resources. In this study, we carried out a large scale of expressed sequence tags (ESTs) sequencing from developing sesame seeds and further conducted analysis on seed storage products-related genes.

Results: A normalized and full-length enriched cDNA library from $5 \sim 30$ days old immature seeds was constructed and randomly sequenced, leading to generation of 41,248 expressed sequence tags (ESTs) which then formed 4,713 contigs and 27,708 singletons with $44.9 \%$ uniESTs being putative full-length open reading frames. Approximately 26,091 of all these uniESTs have significant matches to the counterparts in Nr database of GenBank, and 21,628 of them were assigned to one or more Gene ontology (GO) terms. Homologous genes involved in oil biosynthesis were identified including some conservative transcription factors regulating oil biosynthesis such as LEAFY COTYLEDON1 (LEC1), PICKLE (PKL), WRINKLED1 (WRI1) and majority of them were found for the first time in sesame seeds. One hundred and 17 ESTs were identified possibly involved in biosynthesis of sesame lignans, sesamin and sesamolin. In total, 9,347 putative functional genes from developing seeds were identified, which accounts for one third of total genes in the sesame genome. Further analysis of the uniESTs identified 1,949 nonredundant simple sequence repeats (SSRs).

Conclusions: This study has provided an overview of genes expressed during sesame seed development. This collection of sesame full-length cDNAs covered a wide variety of genes in seeds, in particular, candidate genes involved in biosynthesis of sesame oils and lignans. These EST sequences enriched with full length will contribute to comparative genomic studies on sesame and other oilseed plants and serve as an abundant information platform for functional marker development and functional gene study.
\end{abstract}

\section{Background}

Sesame (Sesamum indicum L.) belonging to the family pedaliaceae [1] is one of the most ancient self-pollinated oilseed crops. Sesame is one of oilseed crops with the highest oil content of up to $62.7 \%$ (average $52 \%$ ) in seeds of sesame cultivars and accessions [2] when seed

\footnotetext{
* Correspondence: liusy@oilcrops.cn

+ Contributed equally

'Key Laboratory for Oil Crops Biology, The Ministry of Agriculture, PR China. Oil Crops Research Institute, Chinese Academy of Agricultural Sciences, No.2 Xudong Second Road, Wuhan 430062, China

Full list of author information is available at the end of the article
}

oil contents of other major oil crops peanut (Arachis hypogaea), oilseed rape (Brassica napus) and soybean (Glycine max) contain up to 54.0\% (average 50\%) [3], up to $46 \%$ (average $39 \%$ ) [4] and up to $27.9 \%$ (average $20 \%$ ) [5], respectively. Although recent intensive selection (imposed on natural variation or hybridization offspring) aided with high throughput Near-infrared spectroscopy for seed oil content determination with no seed damage can lead to more than $50 \%$ in oilseed rape, it is still lower than that having existed in sesame accessions for long time. Furthermore, sesame seed is highly nutritive in protein contents of the seed dry weight $(25 \%)$ and

\section{Biomed Central}


has distinctive flavor. Sesame oil has excellent stability due to the existence of the natural antioxidants such as lignans sesamin, sesamol, sesamolin and sesaminol produced during seed development [6]. These natural antioxidants help to improve health qualities such as inhibiting $\Delta 5$-desaturase in mammals, enhancing vitamin $\mathrm{E}$ bioactivity, and inhibiting proliferation of human cancer cells [7-10]. Sesame lignans may also play a role in sesame resistance to insect pests and microbial pathogens [11].

The study on synthesis of high oil content and these antioxidants is poor, partly because of poor DNA and RNA sequence resources. At present, there are only about 3,000 sesame EST sequences available in GenBank [12]. Lack of sequence information and poor understanding of related synthetic biology have hindered genetic improvement on these economic characters as well as high unsaturated fatty acids contents in sesame. Thereby, our objective in this study was to build a large set of EST collection from developing seeds of sesame.

After construction of a normalized full-length cDNA library, we carried out large scale sequencing of the library and obtained more than 40,000 ESTs from developing seeds of high oil content Chinese sesame cultivar Zhongzhi 14, and further performed bioinformatics analysis on the ESTs with focus on those involved in oil and lignin biosynthesis. These sequence information will serve as the public-accessible fundamental resources for generation of molecular markers, genome annotation, gene discovery in sesame and comparative genomics study on oil contents of major oilseed crops.

\section{Results and discussion EST generation}

To understand accumulation of oil and lignan, and determine time of sampling developing seeds for EST sequencing, two sesame (S. indicum) cultivars Zhongzhi 14 with high oil content and its contrast Miaoqian with low oil content and early maturation were analyzed for accumulation of oil, fatty acids and lignans. The results indicated that the two cultivars have different oil contents but similar profiles of fatty acid compositions (Figure 1). Both cultivars started quick increase in oil contents from 10 days after pollination to maturation and oil accumulation reached their peak at about 25 days. Therefore sampling time was determined as 5, 20 and 30 days after pollination. Considering annual productivity $(1321.5 \mathrm{~kg}$ per hectare of Zhongzhi 14, compared to $425 \mathrm{~kg}$ per hectare of Miaoqian) and oil content of seeds, Zhongzhi 14 was selected for construction of cDNA library enriched in full-length coding sequences [13] and EST sequencing.

The primary titer of the cDNA library was $1 \times 10^{6}$ $\mathrm{cfu} / \mathrm{mL}$ with more than $90 \%$ recombinant clones revealed by X-Gal/IPTG screening and a small-scale quality assessment was performed prior to commencement of large-scale sequencing [13]. Plasmid DNAs were automatically prepared from the cDNA clones and sequenced from the 5' end by the Sanger method.

In total, 41,248 ESTs from single-pass 5' sequencing of 45,569 cDNA clones passed the quality control for high confidence base call with an average read length of approximately $570 \mathrm{bp}$ (Figure 2). The overall sequencing success rate was $91 \%$. The EST sequences generated in this study were deposited in GenBank with the accession numbers JK045130-JK086377. The GC content of the EST sequences was approximately $42.86 \%$. Approximately $32.8 \%$ of the 41,248 sequences appeared twice or more times.

\section{Clustering of ESTs}

After screening of low-quality DNA and trimming of vector sequences, Phrap program [14] was used to cluster the EST sequences and produce a uniESTs data set which comprised 4,713 tentative unique contigs (TUCs, see Additional file 1) and 27,708 tentative unique singletons sequences (TUSs, Table 1). The number of ESTs in
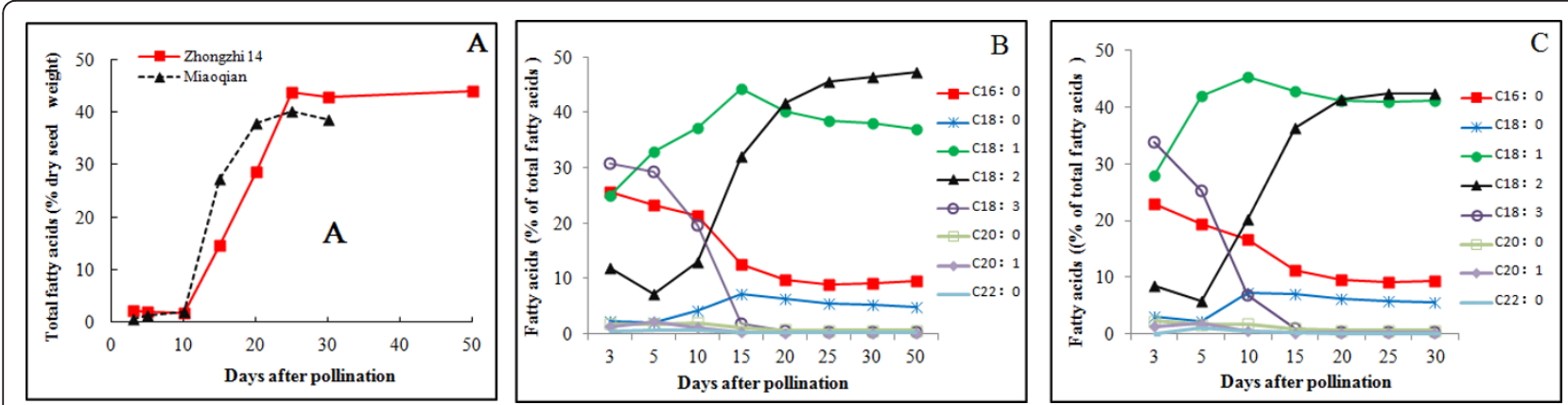

Figure 1 Accumulation of oil and fatty acids in developing seeds of sesame. The seeds of the sesame cultivars (S. indicum) Zhongzhi 14 and Miaoqian grew in two neighboring plots of a field in the institute farm. Fatty acid composition of oil was determined by the GC method. Oil contents (A) were calculated from the total fatty acids contents. B, C: Dynamic changes in fatty acid composition in developing seeds of sesame cultivars Zhongzhi 14 (B) and Miaoqian (C). 


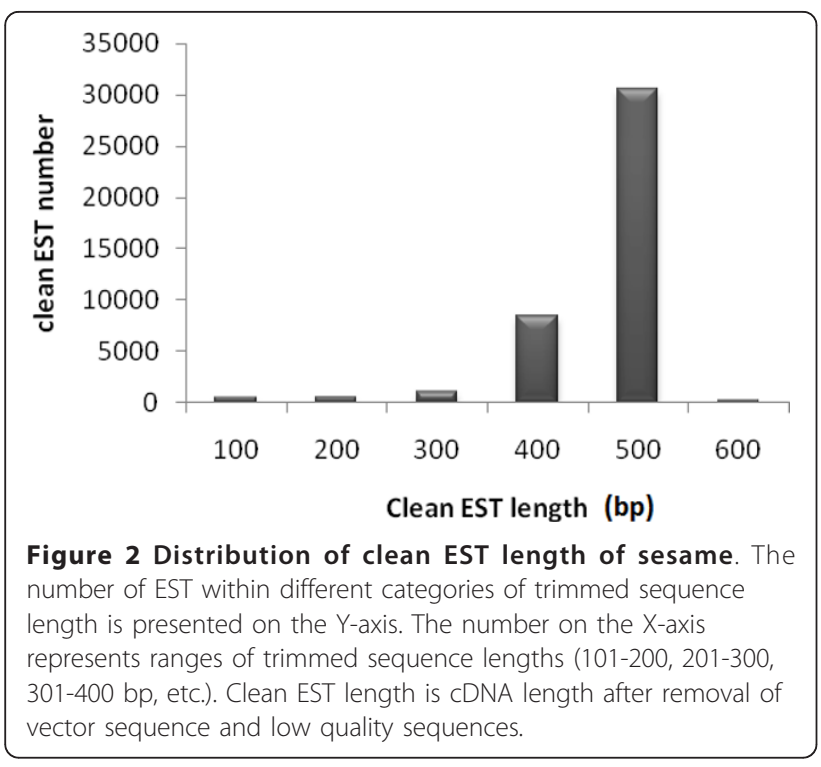

the TUCs ranged from 2 to 92 and there are more than $65.5 \%$ contigs with 2 ESTs, $18.2 \%$ with 3 ESTs and $15.2 \%$ with $4-10$ ESTs.

Among these uniESTs sequences, $80.6 \%$ had significant matches to sequences in the non-redundant protein database $(\mathrm{Nr})$ with an $\mathrm{E}$ value cut-off equal or less than $10^{-5}$. Comparison of our uniESTs data set with the sesame EST sequence in GenBank using BLASTN demonstrated that only 349 uniESTs (1.1\%) in our data set had significant matches to 903 sesame ESTs in GenBank (E-values $\leq 10^{-5}$ ) (Relevant information of these uniESTs was listed in Table 2).

Comparison of the sesame uniESTs against the A. thaliana proteome database using BLASTX indicated $40 \%$ of these uniESTs with significant matches to those from $A$. thaliana (E-values $\leq 10^{-5}$ ). Based on identification of Clusters of Orthologous Groups of protein (COGs) [15] (Figure 3), 10,575 uniESTs (33.0\%) of sesame seeds were assigned to COGs by BLASTX. The proportion pattern of each COG subcategory was similar between sesame and A. thaliana seeds [16] (Figure 3). In these two sets of
cDNA sequences, only 1,360 sesame uniESTs are matched to A. thaliana seed genes (338 genes) [16] and 20\% of these genes were involved in translation, ribosomal proteins synthesis, and $17 \%$ in posttranslational modification, protein turnover, chaperones. Only $1 \%$ of these genes were related to lipid transport and metabolism category.

\section{Protein coding regions}

The full-length open reading frames (ORFs) in the uniESTs data set were identified. Homology search of the 32,421 uniESTs using BLASTX identified uniESTs with relatively high similarity (E-values $\leq 10^{-5}$ ) to known genes, where the sesame sequences, each had a start codon at a position similar to the protein sequence in GenBank, form a data set of putative full-length ORFs (44.9\%). Furthermore, the codon usage table (Table 3 ) for the full-length sesame ORFs was generated using CODONW. The sesame (S. indicum) codon usage table containing 14,669 codons showed the GC content of the predicted coding region (46.7\%) and the GC frequency at the third position (46.4\%). The analysis was the first version of sesame codon usage table which was not presented in the Kazusa codon usage database http://www.kazusa.or.jp/codon/.

\section{Gene ontology annotation}

A total of 18,549 uniESTs were successfully annotated with Gene ontology (GO) terms using Blast2GO program [17]. An additional 3,079 sequences were then annotated using InterProScan program [18]. Overall, a total of 21,628 uniESTs were annotated with 111,600 GO terms distributing among the three main GO categories. 9,347 tentative unique genes (TUGs) representing 21,628 uniESTs across the various GO terms were examined with WEGO [19] (Table 4).

Under the category Biological Process, subcategories "cellular process" and "metabolic process" accounted for approximately $49.5 \%$ and $46.2 \%$ of the annotations for the TUGs, respectively, reflecting activeness of these processes. There are an overlap which is largely represented by the cellular metabolic process (37.1\%) in the two subcategories and in the subcategory "metabolic

Table 1 Summary of ESTs from sesame

\begin{tabular}{llc}
\hline & Number & Percentage of uniESTs \\
\hline Total ESTs sequenced & 45569 & - \\
Number of EST sequences with readable sequence & 41248 & - \\
Redundant sequences & 13540 & - \\
Number of uniESTs & 32421 & - \\
Number of singletons sequences & 27708 & 85.5 \\
Number of tentatively unique contigs & 4713 & 14.5 \\
uniESTs with significant matches against Nr & 26131 & 80.6 \\
uniESTs with non-significant matches against Nr & 6289 & 19.4 \\
uniESTs with significant matches to A. thaliana & 12983 & 40 \\
\hline
\end{tabular}


Table 2 Frequency of sesame ESTs from the present study with significant similarities to sesame genes in the public database

\begin{tabular}{|c|c|}
\hline Putative functions & EST number \\
\hline 11s globulin & 32 \\
\hline Polyubiquitin & 20 \\
\hline tubulin beta-1 chain & 9 \\
\hline alpha tubulin 1 & 7 \\
\hline s-adenosylmethionine synthetase & 7 \\
\hline alanine aminotransferase & 6 \\
\hline rab11-family small gtpase & 6 \\
\hline eukaryotic translation initiation factor $5 \mathrm{a}$ isoform iv & 5 \\
\hline 14-3-3 family protein & 5 \\
\hline Actin & 5 \\
\hline polyubiquitin containing 7 ubiquitin monomers & 4 \\
\hline legumin-like protein & 4 \\
\hline glyceraldehyde 3-phosphate dehydrogenase & 4 \\
\hline aspartic proteinase & 4 \\
\hline asparaginyl endopeptidase rep-2 & 4 \\
\hline nuclear antigen homolog & 4 \\
\hline adenine nucleotide translocator & 4 \\
\hline Calmodulin & 4 \\
\hline $2 s s 1 \_s e s i n 2 s$ seed storage protein 1 precursor (beta-globulin) ( 2 s albumin storage protein) & 4 \\
\hline glutathione s-transferase & 4 \\
\hline kda oleosin & 3 \\
\hline hydroxyproline-rich glycoprotein family protein & 3 \\
\hline acr3 (act domain repeat 3) & 3 \\
\hline heat shock protein 83 & 2 \\
\hline histone h3 & 2 \\
\hline high mobility group protein & 2 \\
\hline 20s proteasome beta subunit & 2 \\
\hline $26 s$ proteasome non-atpase regulatory subunit 14 & 2 \\
\hline $26 s$ proteasome regulatory particle triple-a atpase subunit5a & 2 \\
\hline 3-hydroxy-3-methylglutaryl coenzyme a synthase & 2 \\
\hline 3-ketoacyl-thiolase acetyl-acyltransferase & 2 \\
\hline 40 s ribosomal protein $s 8$ & 2 \\
\hline 60s ribosomal protein 111 & 2 \\
\hline adp-ribosylation factor & 2 \\
\hline alcohol dehydrogenase & 2 \\
\hline ap2 erebp transcription factor & 2 \\
\hline
\end{tabular}

process," primary metabolic process $(36.4 \%)$, macromolecule metabolic process (27\%) and biosynthesis (19.6\%) account for large proportions, suggesting active metabolism of storage substances such as oil, lignan and proteins. In correspondence to these processes, in the main category Molecular Function, $41.8 \%$ of the TUGs annotations were grouped into the subcategory "binding" and $40.2 \%$ in the subcategory "catalytic activity" (Table 4).

\section{Analysis of ESTs involved in oil biosynthesis in developing sesame seeds} Comparative analysis indicated that the most redundant genes related to biosynthesis of fatty acid and oil in sesame included ketoacyl-CoA thiolase (KAT), pyruvate dehydrogenase complex (PDHC), plastidial long-chain acyl-CoA synthetase (LACS), stearoyl-ACP desaturase (SAD), acetylCoA carboxylase (ACC), ketoacyl-CoA reductase (KAR), oil-body oleosin (OBO), diacylglycerol acyltransferase (DGAT) etc. 496 uniESTs candidates were homologous to Arabidopsis oil-related genes (Arabidopsis Lipid Gene Database http://www.plantbiology.msu.edu) [20](Additional file 2). Of these, 71 uniESTs were mapped to the sesame genome sequence (Additional file 3 for DNA sequences of these genes) and just 12 genes like PDHC, $\mathrm{OBO}$ and LACS are homologous to those in the public sesame ESTs database (Additional file 2). 
S. indicum seed

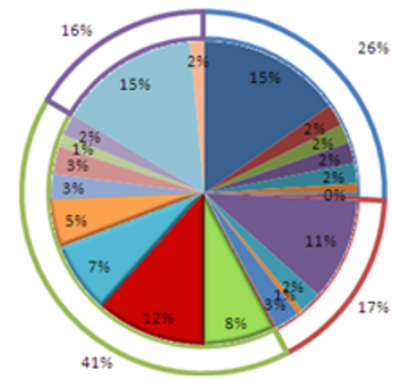

A

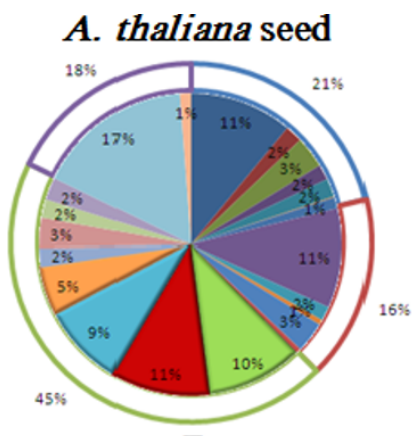

B

\begin{tabular}{|c|c|c|}
\hline & $\begin{array}{l}\text { Posttranslational modification, protein turnover, chaperones } \\
\text { Signal transduction mechanisms }\end{array}$ & $\begin{array}{l}\text { CELLULAR PROCESSES } \\
\text { AND SIGNALING }\end{array}$ \\
\hline & Cell wall/membrane/envelope biogenesis & \\
\hline & Intracellular trafficking, secretion, and vesicular transport & \\
\hline & Cytoskeleton & \\
\hline & Cell cycle control, cell division, chromosome partitioning & \\
\hline 更 & Defense mechanisms & \\
\hline $\mathbf{a}$ & Cell motility & \\
\hline & Nuclear structure & \\
\hline$\square$ & Translation, ribosomal structure and biogenesis & INFORMATION \\
\hline$\square$ & Transcription & STORAGE AND \\
\hline $\mathbf{\square}$ & Chromatin structure and dynamics & PROCESSING \\
\hline घ & Replication, recombination and repair & \\
\hline 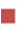 & RNA processing and modification & \\
\hline 回 & Carbohydrate transport and metabolism & METABOLISM \\
\hline ] & Energy production and conversion & \\
\hline 回 & Amino acid transport and metabolism & \\
\hline 田 & Lipid transport and metabolism & \\
\hline घ & Inorganic ion transport and metabolism & \\
\hline 口 & Coenzyme transport and metabolism & \\
\hline II & Secondary metabolites biosynthesis, transport and catabolism & \\
\hline E & Nucleotide transport and metabolism & \\
\hline 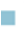 & General function prediction only & POORLY \\
\hline . & Function unknown & CHARACTERIZED \\
\hline
\end{tabular}

Figure 3 Comparison of annotation of sesame ESTs (A) with those of Arabidopsis (B). Two cDNA data sets were classified into functional groups by using the COG database; The colors of each functional group are indicated in the table. Graphs are outlined with multi-color frames which represent four subcategories: 'information storage and processing' (red), 'cellular processing and signaling' (blue), 'metabolism' (green) and 'poorly characterized' (purple). A: S. indicum seed COG annotaiton; B: A. thaliana seed COG annotation.

In the "fatty acid synthesis in the plastids pathway" [20], most of the key enzymes were found in our data set, such as the PDHC, ACC, plastidial acyl carrier protein (ACP) and KAR, except an important enzymes malonyl-CoA: ACP malonyltransferase (MCMT). After blasting against the whole sesame genome assembly, we found that most of these genes had lower copy numbers than those in Arabidopsis, except one gene encoding beta-ketoacyl-ACP synthase I (KAS I) which have 2 copies whereas the genomes of Arabidopsis has 1 copy (see Additional file 2 and Additional file 3). KAS I catalyzes the elongation of fatty acid synthesis for the carbon chain from $\mathrm{C} 4$ to $\mathrm{C} 16$, and played a crucial role in chloroplast division and embryo development [21]. The main components of sesame seed oil, oleic acid and linoleic acid, continuously increased up to about $80 \%$ of total fatty acids in the mature seeds (Figure 1). Some uniESTs related to these fatty acids elongation and desaturation in sesame were first reported in this study, such as putative full-length uniESTs encoding ketoacyl-ACP synthase II (KAS II) SAD and endoplasmic reticulum (ER) oleate desaturase (FAD2) which played important roles in the process of the conversion of 16:0-18:0 and desaturation.

Triacylglycerol (TAG) biosynthesis occurs at the ER and probably involves in reactions in the oil body as well [20]. Only four major genes, DGAT1, phosphatidylcholine: diacylglycerol acyltransferase (PDAT), OBO and caleosin, were detected, which involved in the last step of the TAG synthesis reaction and oil body formation in the pathway of synthesis and storage of oil. DGAT1 and PDAT have been known as the very important genes 
Table 3 Codon usage in S. indicum

\begin{tabular}{|c|c|c|c|c|c|c|c|c|c|c|c|c|c|c|c|c|c|c|c|}
\hline \multirow[b]{3}{*}{ First Letter } & \multirow[b]{3}{*}{ U } & \multicolumn{17}{|c|}{ Second Letter } & \multirow[b]{3}{*}{ Third Letter } \\
\hline & & \multicolumn{4}{|l|}{$\mathrm{U}$} & \multicolumn{4}{|l|}{ C } & \multicolumn{4}{|l|}{$A$} & \multicolumn{5}{|l|}{ G } & \\
\hline & & Phe & UUU & 208 & 0.82 & Ser & UCU & 224 & 1.27 & Tyr & UAU & 192 & 0.67 & Cys & UGU & 113 & 0.9 & $U$ & \\
\hline & & Phe & UUC & 301 & 1.18 & Ser & UCC & 259 & 1.47 & Tyr & UAC & 385 & 1.33 & Cys & UGC & 137 & 1.1 & $\mathrm{C}$ & \\
\hline & & Leu & UUA & 202 & 1.04 & Ser & UCA & 173 & 0.98 & TER & UAA & 49 & 1.20 & TER & UGA & 70 & 1.72 & A & \\
\hline & & Leu & UUG & 298 & 1.53 & Ser & UCG & 108 & 0.61 & TER & UAG & 3 & 0.07 & Trp & UGG & 95 & 1 & $\mathbf{G}$ & \\
\hline & $\bar{C}$ & Leu & CUU & 192 & 0.99 & Pro & CCU & 151 & 0.87 & His & CAU & 151 & 1 & $\operatorname{Arg}$ & CGU & 231 & 1.63 & $U$ & \\
\hline & & Leu & CUC & 216 & 1.11 & Pro & $\mathrm{CCC}$ & 165 & 0.96 & His & CAC & 150 & 1 & Arg & CGC & 180 & 1.27 & $\mathrm{C}$ & \\
\hline & & Leu & CUA & 70 & 0.36 & Pro & CCA & 275 & 1.59 & Gln & CAA & 280 & 1.05 & Arg & CGA & 48 & 0.34 & A & \\
\hline & & Leu & CUG & 190 & 0.98 & Pro & CCG & 100 & 0.58 & Gln & CAG & 254 & 0.95 & Arg & CGG & 72 & 0.51 & G & \\
\hline & $\bar{A}$ & Ile & AUU & 399 & 1.27 & Thr & ACU & 357 & 1.55 & Asn & AAU & 261 & 0.97 & Ser & AGU & 121 & 0.68 & $U$ & \\
\hline & & Ile & AUC & 420 & 1.33 & Thr & ACC & 275 & 1.19 & Asn & $A A C$ & 275 & 1.03 & Ser & AGC & 175 & 0.99 & $C$ & \\
\hline & & Ile & AUA & 126 & 0.4 & Thr & ACA & 212 & 0.92 & Lys & AAA & 520 & 0.99 & Arg & AGA & 222 & 1.57 & A & \\
\hline & & Met & AUG & 375 & 1 & Thr & ACG & 77 & 0.33 & Lys & AAG & 529 & 1.01 & Arg & AGG & 96 & 0.68 & G & \\
\hline & $\bar{G}$ & Val & GUU & 331 & 1.39 & Ala & GCU & 396 & 1.51 & Asp & GAU & 412 & 1.13 & Gly & GGU & 402 & 1.54 & $U$ & \\
\hline & & Val & GUC & 281 & 1.18 & Ala & GCC & 280 & 1.07 & Asp & GAC & 315 & 0.87 & Gly & GGC & 227 & 0.87 & $\mathrm{C}$ & \\
\hline & & Val & GUA & 161 & 0.68 & Ala & GCA & 247 & 0.94 & Glu & GAA & 532 & 1.16 & Gly & GGA & 333 & 1.28 & A & \\
\hline & & Val & GUG & 181 & 0.76 & Ala & GCG & 123 & 0.47 & Glu & GAG & 387 & 0.84 & Gly & GGG & 79 & 0.3 & G & \\
\hline
\end{tabular}

Codon usage was calculated from 122 ORFs containing 14,669 codons.

greatly affecting oil body formation and oil content [22]. $\mathrm{OBO}$ and caleosin were believed to facilitate mobilization of the TAG storage reserves [23,24].

Of 361 (3.9\%) annotated uniESTs with putative transcription factor activity, 8 uniESTs were identified involved in oil accumulation, and they are homologous to $A$. thaliana transcription factor (TF) LEAFY COTYLEDON1 (AtLEC1), PICKLE (AtPKL) and WRINKLED1 (AtWRI1), respectively, suggesting their conservation and importance in transcriptional regulation of the fatty acid biosynthetic pathway. Of these TFs, putative sesame LEC1 (SiLEC1) and SiWRI1, like those in Arabidopsis, are single copies after blasting against whole sesame genome assembly (Additional file 3 for DNA sequences of these genes) and the sequence similarity of the two genes are $47 \%$ and $43 \%$ between sesame and Arabidopsis, respectively (Additional file 4). AtLEC1 positively regulates AtWRI1 and a large repertoire of fatty acid synthetic genes and several glycolytic genes [25]. Overexpression of AtWRI1 in Arabidopsis increased TAG content in transgenic plants [26] and overexpression of AtLEC1 and its orthologs in canola (B. napus) caused an increased fatty acid level in transgenic plants [27]. These homologs identified in sesame may have similar functions in oil biosynthesis.

\section{Analysis of ESTs involved in lignan biosynthesis in developing sesame seeds}

Two major oil-soluble lignans, sesamin and sesamolin [28] were quantitatively determined in the seeds of two cultivars (Zhongzhi 14 and Miaoqian) with contrast oil contents. Total lignan (sesamin and sesamolin) content was detectable 15 days after pollination and continuously accumulated until seed maturation (Figure 4). Interestingly sesamin content continuously increased from 15 days after pollination to seed maturation while sesamolin increased to its highest level at 20 days after pollination, and sesamin content was almost as twice as that of sesamolin at 30 days after pollination (Figure 4). In these two cultivars, it is clear that lignan formation was developmentally regulated $[29,30]-$ lignan accumulation and seed development (maturity) keep the same pace. With seed development, the conversion ratio into sesamolin decreased whereas sesamin accumulation increased (Figure 4).

The antioxidant lignans, sesamin and sesamolin, are biosynthesized via the phenylpropanoid biosynthesis pathway $[29,30]$. In the pathway, tyrosine or phenylalanine is first converted into coniferyl alcohol which then undergoes stereoselective coupling to give pinoresinol, and further pinoresinol is metabolized in maturing seeds to piperitol, sesamin and sesamolin which are catalysed by $\mathrm{O}_{2} / \mathrm{NADPH}$ cytochrome P450s in the reactions 10 , 11 , and 14 where methylenedioxy bridges are formed (Figure 5) [29,30].

One hundred and 17 ESTs, corresponding to 7 EC numbers (EC1.1.1.195, EC 1.14.13.-, EC 1.14.13.11, EC 1.2.1.44, EC 1.6.2.4, EC 2.1.1.68, EC 6.2.1.12) (Figure 5), were identified possibly involved in the biosynthesis of sesame lignans during seed development according to KEGG blast result. Compared to the Arabidopsis database, 94 ESTs were homologous to 32 genes of Arabidopsis. In the GenBank database, there are just 12 sesame ESTs homologous to 4 uniESTs of our dataset 
Table 4 Gene Ontology (GO) classifications of tentative unique genes from sesame according to their involvement in biological process, molecular function and cellular component

\begin{tabular}{|c|c|c|c|}
\hline & uniESTs number & Percentage (\%) & GO \\
\hline \multicolumn{4}{|l|}{ Cellular Component: } \\
\hline Extracellular region & 230 & 2.5 & GO:0005576 \\
\hline Cell & 5202 & 55.7 & GO:0005623 \\
\hline Virion & 20 & 0.2 & GO:0019012 \\
\hline Membrane-enclosed lumen & 273 & 2.9 & GO:0031974 \\
\hline Envelope & 191 & 2.0 & GO:0031975 \\
\hline Macromolecular complex & 999 & 10.7 & GO:0032991 \\
\hline Organelle & 2828 & 30.3 & GO:0043226 \\
\hline Extracellular matrix part & 5 & 0.1 & GO:004442C \\
\hline Extracellular region part & 27 & 0.3 & GO:0044421 \\
\hline Organelle part & 931 & 10.0 & GO:0044422 \\
\hline Virion part & 19 & 0.2 & GO:0044423 \\
\hline Synapse part & 9 & 0.1 & GO:0044456 \\
\hline Cell part & 5056 & 54.1 & GO:0044464 \\
\hline Synapse & 19 & 0.2 & GO:0045202 \\
\hline Symplast & 2 & 0.0 & GO:0055044 \\
\hline \multicolumn{4}{|l|}{ Biological Process: } \\
\hline Developmental process & 1475 & 15.8 & GO:0032502 \\
\hline Reproduction & 339 & 3.6 & GO:0000003 \\
\hline Obsolete biological process & 156 & 1.7 & GO:0008371 \\
\hline Reproductive process & 188 & 2.0 & GO:0022414 \\
\hline Immune system process & 42 & 0.4 & GO:0002376 \\
\hline Response to stimulus & 746 & 8.0 & GO:0050896 \\
\hline Multicellular organismal process & 870 & 9.3 & GO:0032501 \\
\hline Multil-organism process & 151 & 1.6 & GO:0051704 \\
\hline Establishment of localization & 1278 & 13.7 & GO:0051234 \\
\hline Bological adhesion & 35 & 0.4 & GO:0022610 \\
\hline Metabolic process & 4319 & 46.2 & GO:0008152 \\
\hline Rhythmic process & 9 & 0.1 & GO:0048511 \\
\hline Viral reproduction & 17 & 0.2 & GO:0016032 \\
\hline Pigmentation & 1120 & 12.0 & GO:0043473 \\
\hline Locomotion & 29 & 0.3 & GO:0040011 \\
\hline Localization & 1299 & 13.9 & GO:0051179 \\
\hline Growth & 88 & 0.9 & GO:0040007 \\
\hline Cellular process & 4628 & 49.5 & GO:0009987 \\
\hline Cell killing & 1 & 0.0 & GO:0001906 \\
\hline Biological regulation & 1454 & 15.6 & GO:0065007 \\
\hline \multicolumn{4}{|l|}{ Molecular Function: } \\
\hline Transcription regulator activity & 542 & 5.8 & GO:0030528 \\
\hline$C$ atalytic activity & 3759 & 40.2 & GO:0003824 \\
\hline Binding & 3907 & 41.8 & GO:0005488 \\
\hline Antioxidant activity & 24 & 0.3 & GO:0016209 \\
\hline Chaperone regulator activity & 1 & 0.0 & GO:0030188 \\
\hline Translation regulator activity & 102 & 1.1 & GO:0045182 \\
\hline Structural molecule activity & 270 & 2.9 & GO:0005198 \\
\hline Transporter activity & 742 & 7.9 & GO:0005215 \\
\hline
\end{tabular}

encoding three enzyme genes (caffeate O-methyltransferase, COMT; cinnamyl-alcohol dehydrogenase, CAD; two P450 family members). Most of the EST candidates corresponding to enzymatic reactions in the biosynthetic pathway of sesame lignans, such as trans-cinnamate 4monooxygenase $(\mathrm{CA} 4 \mathrm{H})$, oxidoreductases, COMT, 4- 


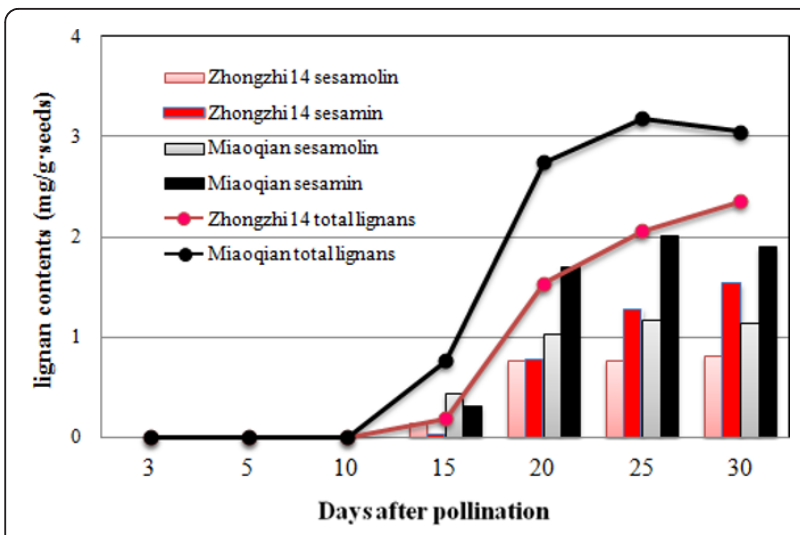

Figure 4 The lignan contents of two sesame cultivars in different development stages. The seeds of the sesame cultivars (S. indicum) Zhongzhi 14 and Miaoqian grew in two neighbouring plots of a field in the institute farm. Lignan content was determined by the HPLC method.

coumarate-CoA ligase (4CL), cinnamoyl-CoA reductase (CCR), CAD etc., were first identified from sesame (Additional file 5). The gene 4CL has the most abundant
ESTs (45 putative uniESTs). There are 5 copies of the gene encoding COMT in the sesame genome (Additional file 6 for DNA sequences of these genes), but this gene is absent in the genomes of Arabidopsis and soybean. Surprisingly, neither phenylalanine ammonia-lyase nor tyrosine ammonia-lyase encoded ESTs were detected in the sesame seed ESTs. Thirty five ESTs of putative NADPH-cytochrome P450 oxidoreductase were identified, which involve in the important steps of sesame lignan production, including a gene encoding CYP81Q1 known for dual methylendioxy bridge formation on pinoresinol to produce sesamin via piperitol [31]. Because little information is available about enzymes for reactions from sesamin to sesamolin and from piperitol to sesamolinol [32], ESTs responsible for these enzymes was not able to be identified.

\section{EST-derived simple sequence repeat (SSR) markers}

Simple-sequence repeats (SSRs) have become one of major markers for population genetic analyses and marker-aided breeding [33,34]. A shortage of sesame molecular markers especially the EST-derived SSRs (EST-

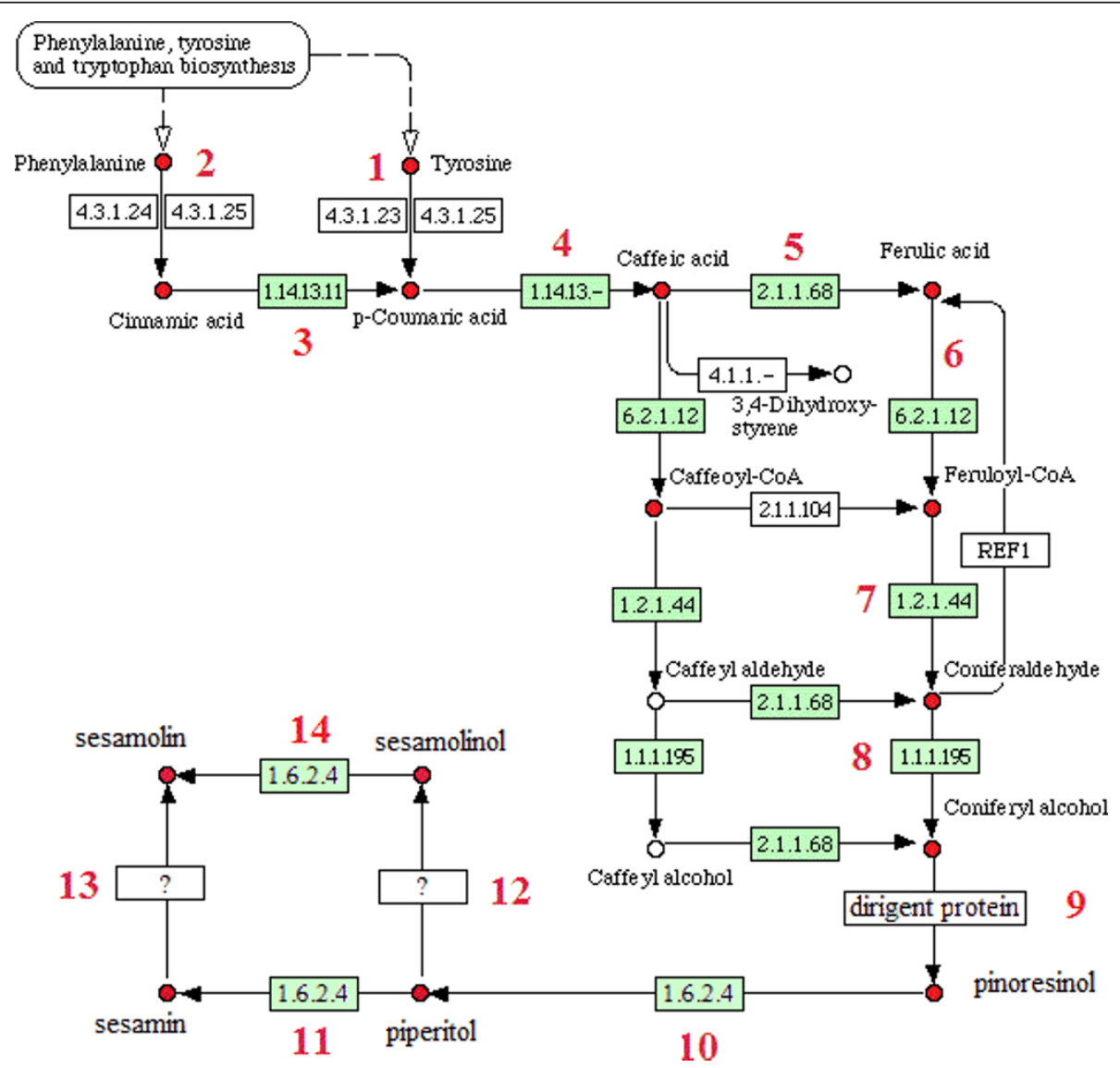

Figure 5 Possible biosynthetic pathways for sesame lignans, sesamin and sesamolin. The pathway map was constructed based on KEGG pathway map (see http://www.genome.jp/kegg-bin/show_pathway?map00940). Numbers correspond to individual reactions and serve to identify the respective enzyme in Additional file 5. Candidate gene found in the present sesame seed ESTs dataset was shown as green. 
SSRs) have limited the efficiency of sesame molecular breeding [35]. Hence development of a large collection of EST-SSR will be very important source. In total, 1,688 uniESTs containing 1,949 non-redundant (NR) SSRs were identified from 32,421 uniESTs. The uniESTs represented about $17.428 \mathrm{Mb}$ of genic sequences. A total of 226 sequences contain more than one SSR. The EST-derived NR SSRs were represented by mono-, di-, tri-, tetra- and pentanucleotide repeat motifs. This corresponds to an overall SSR density of approximately one SSR per $8.9 \mathrm{~kb}$ or one SSR-containing sequence in $5.2 \%$ of the NR EST sequences. About $8.3 \%$ of the SSRs identified were compound SSRs, which were defined as two neighbouring repeats located less than 10 nucleotides apart in a single sequence. The frequencies of the SSR motifs identified from 32,421 uniESTs were summarized in Table 5. Based on the distribution of SSR motifs, AG/ CT motifs represented the most abundant dinucleotide repeat motifs (about 68\%). The most common trinucleotide repeat motifs are, AAG/CTT (21\%) and the most abundant tetranucleotide repeat motifs are the AAGT/ ATTC (13\%) (Figure 6).

\section{Conclusion}

This study provided a set of ESTs enriched in full-length coding sequence generated from developing seeds. The number of these ESTs is more than 12.4 fold of the total number of entries for sesame in GenBank. From this set of ESTs, 9,347 putative functional genes from developing seeds were identified, accounting for one third of total genes in the genome. Most of the transcripts are the first representatives from sesame seeds. Most key enzymes and regulatory factors involved in fatty acid metabolism were found in our data, especially the enzymes responsible for the main unsaturated fatty acid synthesis, TAG synthesis reactions and oil body formation. Some conservative TFs significantly regulating oil synthesis were also identified. These provide a foundation for future comparative analysis of oil biosynthesis of different oilseeds. The uniESTs associated with biosynthetic enzymes of sesame lignans, sesamin and sesamolin, were identified and this information will be helpful for further studies on sesame lignan production. This large number of ESTs, half of them with full-length, have been used for sesame genome annotation in our sesame genome sequencing project and will be useful resource for the functional gene analysis and molecular marker development of traits such as contents of oil, fatty acids and lignan in sesame and for comparative genomics study of seed oil biosynthesis in different oilseed plants.

\section{Methods}

\section{Plant materials}

The seeds of the sesame cultivars (S. indicum) Zhongzhi 14 and Miaoqian from the Oil Crops Research Institute,
Chinese Academy of Agricultural Sciences were sowed in two neighbouring plots of a field in the institute farm. Flowers were tagged in the early morning of the flower opening days. Developing capsules were harvested at 5-50 days or 30 days for early mature Miaoqian after pollination and seeds were immediately isolated and frozen in liquid nitrogen for RNA extraction or dried for seed chemical determination. Frozen seeds were stored at $-70^{\circ} \mathrm{C}$ until use.

\section{Method for determination of sesame seed fatty acid}

Fresh sesame seeds isolated from capsules were dried and ground for solvent extraction (soxlhet method). Fatty acid composition of oil was determined by the GC method according to the National Standards methods of China GB14489.3-1993-T, GBT 17376-2008, and GBT17377-2008 (the Official Methods and Recommended Practices of China). Heptadecanoic acid (17:0) was added to each sample as an internal standard. Oil contents were calculated from the total fatty acids contents.

\section{HPLC analysis of lignans}

Lignans were determined by the HPLC method according to the National Standards methods of China NY/T 1595-2008. Sesamol was used as internal standards for calculating the percentage recovery of lignans.

\section{RNA extraction}

Total RNA was isolated from developing seeds of sesame (cv. Zhongzhi 14) 5, 20, 30 days after pollination, respectively, and mixed in the same proportion, with TRIzol reagent (Gibco-BRL) according to the manufacturer's instruction. Poly A + RNA isolation was performed using PolyA Tract Isolation System (Promega, USA) according to the manufacturers' instructions.

\section{Generation of ESTs}

Construction of a normalized cDNA library enriched in full-length sequences was reported [13]. The titer of unamplified cDNA library was about $1.0 \times 10^{6} \mathrm{cfu} / \mathrm{mL}$. The percentage of recombinants was $100 \%$. The results of gel electrophoresis showed fragments ranged from $700 \mathrm{bp}$ to $2,000 \mathrm{bp}$, with an average length of $1,800 \mathrm{bp}$. The cDNA clones were cultured for plasmid DNA preparation manually. Automated cycle sequencing was performed by the Sanger method using T3 universal primer and BigDye Terminator (Applied Biosystems, USA) or ET Terminator (Amersham Pharmacia Bioscience, USA).

\section{Clustering analysis and annotation}

Quality control of raw DNA sequences was performed by using Phred program [14]http://www.phrap.org/ 
Table 5 Frequency of non-redundant uniESTs-derived SSR motifs

\begin{tabular}{|c|c|c|c|c|c|c|c|c|c|c|c|c|c|c|}
\hline Repeats & 4 & 5 & 6 & 7 & 8 & 9 & 10 & 11 & 12 & 13 & 14 & 15 & $>15$ & total \\
\hline $\mathrm{AC/GT}$ & - & - & 36 & 15 & 11 & 9 & 6 & 2 & 1 & & & & & 80 \\
\hline $\mathrm{AG} / \mathrm{CT}$ & - & - & 99 & 54 & 35 & 25 & 23 & 15 & 6 & 5 & 6 & 2 & 5 & 275 \\
\hline AT/AT & - & - & 28 & 9 & 7 & 3 & & & & & & & & 47 \\
\hline $\mathrm{CG} / \mathrm{CG}$ & - & - & 1 & & & & & & & & & & & 1 \\
\hline AAC/GTT & 217 & 40 & 13 & 3 & & & & & 1 & & & & & 274 \\
\hline $\mathrm{AAG} / \mathrm{CT}$ & 240 & 31 & 9 & 4 & 1 & 3 & & 3 & 1 & 3 & 1 & & 2 & 298 \\
\hline AAT/ATT & 74 & 13 & 2 & 1 & & & & & & & & & & 90 \\
\hline ACC/GGT & 134 & 22 & 13 & 4 & 4 & 1 & & & & & & & & 178 \\
\hline ACG/CTG & 77 & 32 & 3 & 7 & 2 & & 1 & & & & & & & 122 \\
\hline ACT/ATG & 53 & 8 & 6 & 8 & & & & & & & & & & 75 \\
\hline AGC/CGT & 108 & 18 & 12 & 3 & 8 & 1 & & & & & & & & 150 \\
\hline $\mathrm{AGG} / \mathrm{CCT}$ & 91 & 22 & 3 & & 1 & 1 & & & & & & & & 118 \\
\hline AGT/ATC & 31 & 18 & 3 & & & 2 & 3 & & & & & & 1 & 58 \\
\hline CCG/CGG & 53 & 23 & 3 & & 1 & & & & & & & & & 80 \\
\hline $\mathrm{AAAG} / \mathrm{CTT}$ & 5 & 1 & 1 & 1 & & & & & & & & & & 8 \\
\hline AAAT/ATTT & 6 & & & & & & & & & & & & & 6 \\
\hline AACC/GGTT & 8 & & & & & & & & & & & & & 8 \\
\hline AACG/CTTG & 1 & 1 & & 1 & & & & & & & & & & 3 \\
\hline AACT/ATTG & 1 & & & & & & & & & & & & & 1 \\
\hline AAGC/CGTT & & & 1 & & & & & & & & & & & 1 \\
\hline AAGG/CCTT & & 5 & & & & & & & & & & & & 5 \\
\hline AAGT/ATTC & 12 & & & & & & & & & & & & & 12 \\
\hline AATC/AGTT & 1 & & & & & & & & & & & & & 1 \\
\hline AATT/AATT & 1 & & & & & & & & & & & & & 1 \\
\hline ACAG/CTGT & 5 & & 1 & & & & & & & & & & & 6 \\
\hline ACAT/ATGT & 2 & & 5 & & & & & & & & & & & 7 \\
\hline ACGC/CGTG & 3 & & & & & & & & & & & & & 3 \\
\hline ACGT/ATGC & 1 & & & & & & & & & & & & & 1 \\
\hline ACTC/AGTG & 4 & 2 & 2 & & & & & & & & & & & 8 \\
\hline ACTG/ACTG & & 2 & & & & & & & & & & & & 2 \\
\hline AGAT/ATCT & 2 & & & & & & & & & & & & & 2 \\
\hline AGCC/CGGT & & & 1 & 1 & & & & & & & & & & 2 \\
\hline AGGT/ATCC & 6 & 2 & 1 & 1 & & & & & & & & & & 10 \\
\hline AGTC/AGTC & 2 & & & & & & & & & & & & & 2 \\
\hline AGAGG/CCTCT & - & & 5 & & & & & & & & & & & 5 \\
\hline AGGGT/ATCCC & - & 2 & & & & & & & & & & & & 2 \\
\hline AACAAG/CTTGTT & - & & 1 & & & & & & & & & & & 1 \\
\hline AAGTAG/ATCTTC & - & 1 & 1 & & & & & & & & & & & 2 \\
\hline ACCGAG/CTCTGG & - & & & & & & & & & & & & 1 & 1 \\
\hline AGCTCC/AGGTCG & - & 1 & & & & & & & & & & & & 1 \\
\hline
\end{tabular}

Non-redundant SSR motifs were derived from 32,421 uniESTs.

phredphrapconsed.html to remove sub-standard reads, the vector and adapter sequences, followed by EST-trimmer http://pgrc.ipk-gatersleben.De/misa/download/est_trimmer.pl to eliminate 3' polyA and 100 bp EST reads. Phrap program was used to cluster the overlapping ESTs into contigs. Groups that contained only one sequence were classified as singletons. The edited EST was translated into six reading frames and compared with the non-redundant protein database at the National
Center for Biotechnology Information (NCBI) and the Arabidopsis thaliana Database at The Arabidopsis Information Resource (TAIR) http://www.arabidopsis.org/ using BLASTX program with the default settings (NCBI, ftp://ftp.ncbi.nlm.nih.gov/blast). BLASTN program was used to compare our nucleotide sequences with the sequences in the EST database in GenBank. BLASTX and BLASTN results with E-values cut-off $1 \mathrm{e}-10^{-5}$ were treated as 'significant matches,' whereas ESTs with no 


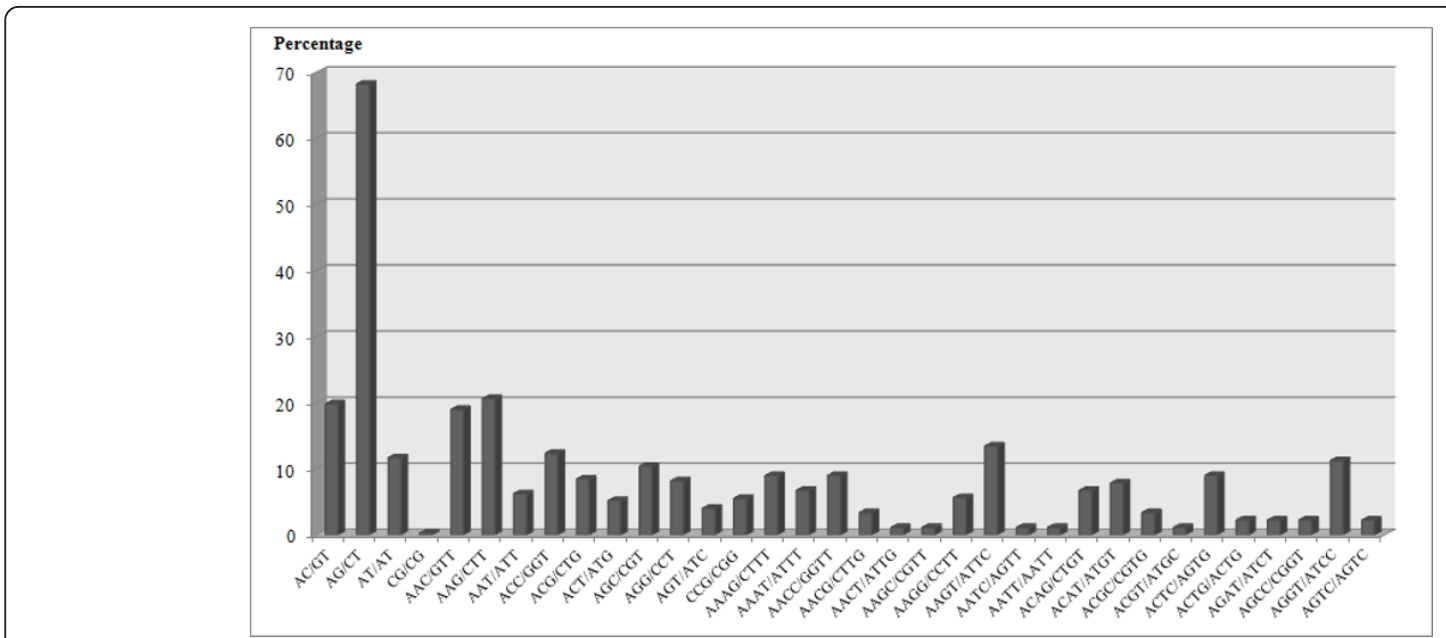

Figure 6 The percentage distribution of the different SSR motifs (mono-, di-, tri-, tetra- and pentanucleotide).

hits or matches with E-values more than $10^{-5}$ to proteins in GenBank were classified as 'no significant matches'.

\section{Assignment of $\mathrm{GO}$ terms}

Gene Ontology (GO) terms were assigned to UniESTs by using Blast2GO [17] and summarized according to their molecular functions, biological processes and cellular components. The software performed BLASTX similarity search against the GenBank non-redundant protein $(\mathrm{Nr})$ database, retrieved $\mathrm{GO}$ terms for the top 12 BLAST results and annotated the sequences based on defined criteria. A weightage based on the default Evidence Code Weights was also used to determine the GO terms annotated. In order to increase the number of sequences annotated by GO terms, additional information and GO terms were obtained by comparing the sequences to the InterPro database using the InterProScan tool [18]. More detailed functional annotations were performed by mapping tentative unique genes (TUGs) to the Gene Ontology Consortium structure which provided a structured and controlled vocabulary to describe gene products according to three ontologies: cellular components, biological processes and molecular functions. The distribution and percentage of TUGs in each of the GO terms were calculated. The GO terms were compared and visualized using WEGO http://wego.genomics.org. $\mathrm{cn}[19]$. The percentage of 100 was defined as the total number of TUGs that were assigned GO terms. However, the percentages of the subcategories did not add up to $100 \%$ because many genes were involved in different classes of function and therefore annotated by multiple GO terms.

\section{Additional material}

Additional file 1: Tentative unique contigs of sesame

Additional file 2: A list of uniESTs involved in lipid metabolism and relevant homologies of Arabidopsis and sesame genes

Additional file 3: The blat and blast result of the uniESTs involved in lipid metabolism to $S$. indicum genome sequence and putative genes.

Additional file 4: Phylogenetic analysis of the two transcriptional factor WRI1 and LEC1 Proteins. The phylogenetic tree was built by MEGA5 [36].

Additional file 5: The blat [37] and blast result of the uniESTs involved in lignans metabolism to $\mathrm{S}$. indicum genome sequence and putative genes.

Additional file 6: Candidate ESTs involved in biosynthetic pathways for sesame lignans, sesamin and sesamolin, and relevant homologies of Arabidopsis and soybean genes.

\section{Acknowledgements}

This work was financially supported by grants from the China National Basic Research Program (2011CB109300), the genetically modified organisms breeding major projects (2009ZX08004-002B), the Open Project of Key Laboratory for Oil Crops Biology, the Ministry of Agriculture, PR China (200703), and the Core Research Budget of the Non-profit Governmental Research Institution. The cDNA library sequencing was conducted by BGIBeijing.

\section{Author details}

${ }^{1}$ Key Laboratory for Oil Crops Biology, The Ministry of Agriculture, PR China. Oil Crops Research Institute, Chinese Academy of Agricultural Sciences, No.2 Xudong Second Road, Wuhan 430062, China. ${ }^{2}$ Department of Life Science and Technology, Nanyang Normal University, Wolong Road, Nanyang 473061, China.

\section{Authors' contributions}

SYL contributed to the conception, design and coordination of the study. HM and CHD were involved in the generation of sesame ESTs. TK analyzed the data and drafted the manuscript and SYL revised the manuscript. HC and XYD conducted determination of sesame seed fatty acids. YZZ and HYL prepared sesame cultivars materials. CBT assembled the sesame genome 
sequence and involved in the analysis of the sequence. All authors have read and approved the final manuscript.

Received: 20 August 2011 Accepted: 24 December 2011

Published: 24 December 2011

\section{References}

1. Dorothea B: Evolution of sesame revisited: domestication, diversity and prospects. Gene Resour Crop Ev 2003, 50(7):779-787.

2. Arslan Ç, Uzun B, Ülger S, İlhan Çağırgan M: Determination of oil content and fatty acid composition of sesame mutants suited for intensive management conditions. J Am Oil Chem Soc 2007, 84(10):917-920.

3. Raheja RK, Batta SK, Ahuja KL, Labana KS, Singh M: Comparison of oil content and fatty acid composition of peanut genotypes differing in growth habit. Plant Food Hum Nutr (Formerly Qualitas Plantarum) 1987, 37(2):103-108.

4. Li RJ, Wang HZ, Mao H, Lu YT, Hua W: Identification of differentially expressed genes in seeds of two near-isogenic Brassica napus lines with different oil content. Planta 2006, 224(4):952-962.

5. Seed Composition. Edited by: Wilson RF. Madison: American Society of Agronomy, Crop Science Society of America, and Soil Science Society of America; , 3 2004:

6. Shyu Y-S, Hwang LS: Antioxidative activity of the crude extract of lignan glycosides from unroasted Burma black sesame meal. Food Res Int 2002, 35(4):357-365.

7. Cooney RV, Custer LJ, Okinaka L, Franke AA: Effects of dietary sesame seeds on plasma tocopherol levels. Nutr Cancer 2001, 39(1):66-71.

8. Kang MH, Naito M, Sakai K, Uchida K, Osawa T: Mode of action of sesame lignans in protecting low-density lipoprotein against oxidative damage in vitro. Life Sci 2000, 66(2):161-171

9. Miyahara $Y$, Hibasami H, Katsuzaki H, Imai K, Komiya T: Sesamolin from sesame seed inhibits proliferation by inducing apoptosis in human lymphoid leukemia Molt 4B cells. Int J Mol Med 2001, 7(4):369-371.

10. Shimizu S, Akimoto K, Shinmen Y, Kawashima H, Sugano M, Yamada H: Sesamin is a potent and specific inhibitor of $\Delta 5$ desaturase in polyunsaturated fatty acid biosynthesis. Lipids 1991, 26(7):512-515.

11. Harmatha J, Nawrot J: Insect feeding deterrent activity of lignans and related phenylpropanoids with a methylenedioxyphenyl (piperonyl) structure moiety. Entomol Exp App/ 2002, 104(1):56-60.

12. Suh MC, Kim MJ, Hur CG, Bae JM, Park Yl, Chung CH, Kang CW Ohlrogge JB: Comparative analysis of expressed sequence tags from Sesamum indicum and Arabidopsis thaliana developing seeds. Plant Mol Biol 2003, 52(6):1107-1123.

13. Ke T, Dong $\mathrm{CH}$, Mao H, Zhao YZ, Liu HY, Liu SY: Construction of a normalized full-length cDNA library of sesame developing seed by DSN and SMART. Agricultural Sciences in China 2011, 10(7):1004-1009.

14. Gordon D, Abajian C, Green P: Consed: a graphical tool for sequence finishing. Genome Res 1998, 8(3):195-202.

15. Tatusov RL, Fedorova ND, Jackson JD, Jacobs AR, Kiryutin B, Koonin EV, Krylov DM, Mazumder R, Mekhedov SL, Nikolskaya AN, Rao BS, Smirnov S, Sverdlov AV, Vasudevan S, Wolf YI, Yin JJ, Natale DA: The COG database: an updated version includes eukaryotes. BMC Bioinformatics 2003, 4:41

16. White JA, Todd J, Newman T, Focks N, Girke T, de llarduya OM, Jaworski JG, Ohlrogge JB, Benning C: A new set of Arabidopsis expressed sequence tags from developing seeds. The metabolic pathway from carbohydrates to seed oil. Plant Physiol 2000, 124(4):1582-1594.

17. Conesa A, Gotz S, Garcia-Gomez JM, Terol J, Talon M, Robles M: Blast2GO: a universal tool for annotation, visualization and analysis in functional genomics research. Bioinformatics 2005, 21(18):3674-3676.

18. Zdobnov EM, Apweiler R: InterProScan-an integration platform for the signature-recognition methods in InterPro. Bioinformatics 2001, 17(9):847-848.

19. Ye J, Fang L, Zheng H, Zhang Y, Chen J, Zhang Z, Wang J, Li S, Li R, Bolund L, Wang J: WEGO: a web tool for plotting GO annotations. Nucleic Acids Res 2006, 34(Web Server):W293-297.

20. Li-Beisson Y, Shorrosh B, Beisson F, Andersson MX, Arondel V, Bates PD, Baud S, Bird D, DeBono A, Durrett TP, Franke RB, Graham IA, Katayama K, Kelly AA, Larson T, Markham JE, Miquel M, Molina I, Nishida I, Rowland O, Samuels L, Schmid KM, Wada H, Welti R, Xu C, Zallot R, Ohlrogge J: AcylLipid Metabolism. The Arabidopsis Book 2010, 8:e0133.
21. Wu GZ, Xue HW: Arabidopsis beta-ketoacyl-[acyl carrier protein] synthase $\mathrm{i}$ is crucial for fatty acid synthesis and plays a role in chloroplast division and embryo development. Plant Cell 2010, 22(11):3726-3744.

22. Zhang M, Fan J, Taylor DC, Ohlrogge JB: DGAT1 and PDAT1 acyltransferases have overlapping functions in Arabidopsis triacylglycerol biosynthesis and are essential for normal pollen and seed development. Plant Cell 2009, 21(12):3885-3901.

23. Poxleitner M, Rogers SW, Lacey Samuels A, Browse J, Rogers JC: A role for caleosin in degradation of oil-body storage lipid during seed germination. Plant J 2006, 47(6):917-933.

24. Shimada TL, Shimada T, Takahashi H, Fukao Y, Hara-Nishimura I: A novel role for oleosins in freezing tolerance of oilseeds in Arabidopsis thaliana. Plant J 2008, 55(5):798-809.

25. Mu J, Tan H, Zheng Q, Fu F, Liang Y, Zhang J, Yang X, Wang T, Chong K, Wang XJ, Zuo J: LEAFY COTYLEDON1 is a key regulator of fatty acid biosynthesis in Arabidopsis. Plant Physiol 2008, 148(2):1042-1054.

26. Cernac A, Benning C: WRINKLED1 encodes an AP2/EREB domain protein involved in the control of storage compound biosynthesis in Arabidopsis. Plant J 2004, 40(4):575-585

27. Tan H, Yang X, Zhang F, Zheng X, Qu C, Mu J, Fu F, Li J, Guan R, Zhang H, Wang G, Zuo J: Enhanced seed oil production in canola by conditional expression of Brassica napus LEAFY COTYLEDON1 and LEC1-LIKE in developing seeds. Plant Physiol 2011, 156(3):1577-1588.

28. Mitsuo N: The chemistry and physiological functions of sesame. Food Reviews International 1995, 11(2):281-329.

29. Jiao $Y$, Davin LB, Lewis NG: Furanofuran lignan metabolism as a function of seed maturation in Sesamum indicum: methylenedioxy bridge formation. Phytochemistry 1998, 49(2):387-394.

30. Kato MJ, Chu A, Davin LB, Lewis NG: Biosynthesis of antioxidant lignans in Sesamum indicum seeds. Phytochemistry 1998, 47(4):9.

31. Ono E, Nakai M, Fukui $Y$, Tomimori N, Fukuchi-Mizutani M, Saito M, Satake H, Tanaka T, Katsuta M, Umezawa T, Tanaka Y: Formation of two methylenedioxy bridges by a Sesamum CYP81Q protein yielding a furofuran lignan, (+)-sesamin. Proc Natl Acad Sci USA 2006, 103(26):10116-10121.

32. Marchand PA, Zajicek J, Lewis NG: Oxygen insertion in Sesamum indicum furanofuran lignans. diastereoselective syntheses of enzyme substrate analogues. Can J Chem 1997, 75(6):840-849.

33. Ellis JR, Burke JM: EST-SSRs as a resource for population genetic analyses. Heredity 2007, 99(2):125-132.

34. Varshney RK, Graner A, Sorrells ME: Genic microsatellite markers in plants: features and applications. Trends Biotechnol 2005, 23(1):48-55.

35. Zhang HY, Wei LB: Development and utilization of EST-derived microsatellites in sesame (Sesamum indicum L.). ACTA Agronomica Sinica 2008, 34(12):2077.

36. Tamura K, Peterson D, Peterson N, Stecher G, Nei M, Kumar S: MEGA5: molecular evolutionary genetics analysis using maximum likelihood, evolutionary distance, and maximum parsimony methods. Mol Biol Evol 2011, 28(10):2731-2739.

37. Kent WJ: BLAT-the BLAST-like alignment tool. Genome Res 2002, 12(4):656-664

doi:10.1186/1471-2229-11-180

Cite this article as: Ke et al:: Analysis of expression sequence tags from a full-length-enriched cDNA library of developing sesame seeds (Sesamum indicum). BMC Plant Biology 2011 11:180. 